\title{
Prediction Method for Wrinkle Grade by Image Analysis
}

\author{
MATSUOKA Toshio a," ${ }^{\text {, }}$ KANAI Hiroyuki ${ }^{\mathrm{b}}$, KIMURA Hirokazu ${ }^{\mathrm{c}}$, KOUSO Takeyoshi ${ }^{\mathrm{b}}$, \\ SHIBATA Kiyohiro ${ }^{\mathrm{d}}$, NISHIMATSU Toyonori ${ }^{\mathrm{b}}$ \\ ${ }^{a}$ Mie Prefecture Industrial Research Institute, 5-5-45 Takajaya, Tsu, Mie 514-0819, Japan \\ ${ }^{\mathrm{b}}$ Faculty of Textile Science and Technology, Shinshu University, 3-15-1 Tokida, Ueda, Nagano 386-8567, Japan \\ ${ }^{\mathrm{c}}$ Technology Research Institute of Osaka Prefecture, 2-7-1 Ayumino, Izumi, Osaka 594-1157, Japan \\ d AOKI Inc., 60-1 Meichi Shimoyanaginouchi, Ichinomiya, Aichi 494-0012, Japan
}

Received 15 July 2008; accepted for publication 16 September 2008

\begin{abstract}
This paper describes an objective evaluation method for fabric wrinkles of shirts formed after washing. The fabric wrinkle images were measured by texture analysis and evaluated subjectively by experts. The textural features of wrinkle images have a significant correlation with wrinkle grades evaluated by experts. A principal component analysis with textural features of images was carried out, and also a multiple regression analysis was carried out using the two principle component scores as explanatory variables and wrinkle grades evaluated by experts as criterion variables. As the result, we proposed an objective evaluation equation for predicting wrinkle grades. It was found that the wrinkle grades predicted by the equation have a significant correlation with the result of subjective evaluation, and the fabric wrinkles of shirts after washing is objectively evaluated with our proposed equation.
\end{abstract}

Key Words: Wrinkle after washing, Wrinkle grade, Sensory evaluation, Textural analysis

\section{Introduction}

Wrinkles in clothes are usually formed by being worn, after washing, or by being folded for storage. Wrinkles are one of the important factors for the appearance evaluation of clothes. In recent years, consumers have become interested in the wrinkle-resistance of clothes, and there are many wrinkle resistant shirts on the markets. A lot of studies on wrinkle-resistance have been conducted, and an objective method to evaluate fabric wrinkles has always been sought.

Methods utilizing wrinkle testers [1] or methods utilizing comparison with replicas [2] are well known as evaluation methods of wrinkle recovery of fabric. These evaluation methods are influenced by the psychology / physiology of a subject and the testing environment. Various other methods to evaluate wrinkles objectively have been examined by other researchers [3-11].

Some papers have discussed 3-D measurement of the wrinkle replicas by the finite difference method [3], the slit beam projecting technique [4], the CCD laser displacement sensor [5], or using the projecting grid technique [6]. These papers concluded that 3-D measurement of the replicas and their analysis had been effective for objective evaluation.
However, this method used replicas and did not examine correspondence with real fabric wrinkles formed by being worn or washing.

Objective methods for evaluating wrinkle appearances using image analysis were examined [7-10]. It is well known that the image information supplied by texture analysis is useful for evaluating fabric wrinkles. However, it does not examine correlations between the wrinkle grades evaluated by proposal methods and the grades evaluated by experts. Only one study [11] has examined these correlations, and this study concluded that fabric wrinkles of trousers formed by being worn have been predicted by the degree of similarity between images. However the accuracy of the method is a little low. This means that no research has yet been carried out to develop a prediction method for fabric wrinkles of shirts formed by washing.

Our research provides an objective evaluation method for fabric wrinkles of shirts formed by washing. The fabric wrinkle images were measured by texture analysis and evaluated subjectively by experts. We proposed an objective evaluation equation of wrinkle grades using the principal component analysis and the multiple regression analysis.

* Corresponding author: E-mail: matsut25@pref.mie.jp, Tel: +81-59-234-8468, Fax: +81-59-234-3982 


\section{Experimental method}

\section{1 Fabric samples}

Fabric samples were three plain woven fabrics, these fiber contents were $100 \%$ cotton, $50 \%$ cotton $50 \%$ polyester blend, or $15 \%$ cotton $85 \%$ polyester blend. Yarn counts for both warp and weft yarns were 50s and threads in fabric were 144 ends/inch and 76 picks/inch. Samples were sewn in a cylindrical shape. The length of the sample was $325 \mathrm{~mm}$, the width of the top was $111 \mathrm{~mm}$, and the width of bottom was $89 \mathrm{~mm}$.

\section{2 Wrinkling device and forming wrinkles}

We made a wrinkling device (shown in Fig. 1(a)) which forms wrinkles by tucking a fabric sample into a case. This

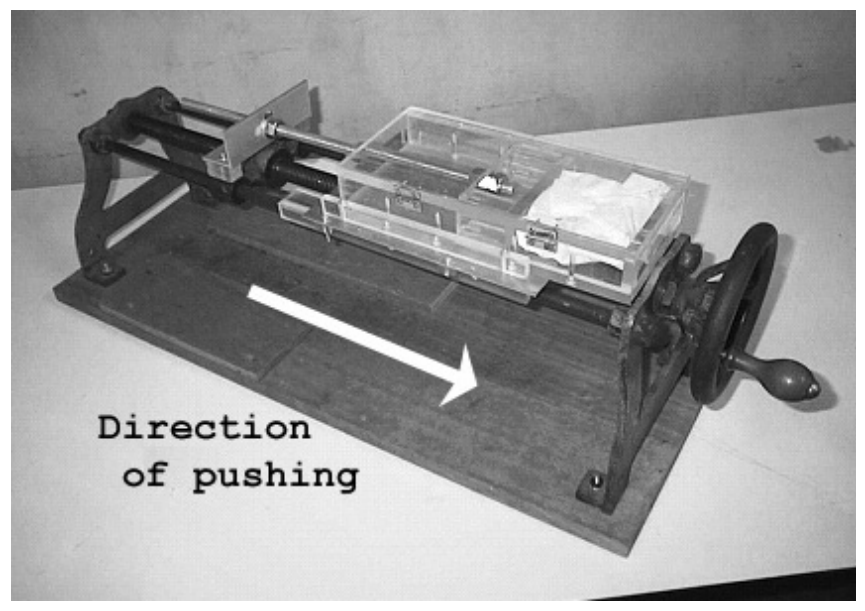

(a) Photograph of the device

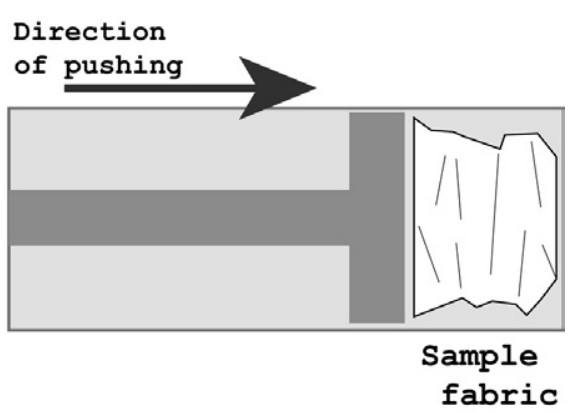

(b) Outline of the case

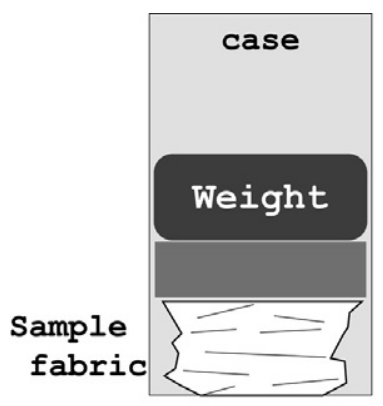

(c) Forming wrinkles

Fig. 1 Wrinkling device.

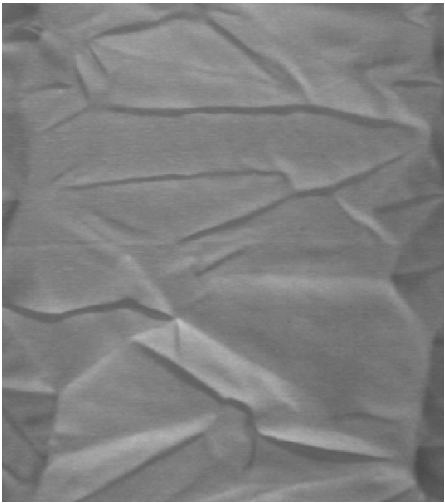

Fig. 2 Fabric wrinkle image (grade 2) formed by the wrinkling device.

device forms wrinkles which imitate wrinkles formed on sleeves of shirts. The characteristic of this device is as follows. As a cylindrical sample was used, this device could form the wrinkle which resembled a real wrinkle on sleeves of shirts than that formed by the wrinkle tester [1]. And an installation method of the sample in this device was easier than that in the wrinkle tester [1].

First, a fabric sample was forced into a case $(250 \mathrm{~mm}$ long, $100 \mathrm{~mm}$ wide, $30 \mathrm{~mm}$ high) of the wrinkling device (shown in Fig. 1(b)). Then, the case was taken off from the device and raised. After that, forming various grades of wrinkles, and various loads of $0 \mathrm{~N}, 14.7 \mathrm{~N}, 24.5 \mathrm{~N}$, and $39.2 \mathrm{~N}$ were put individually on the sample in the case (shown in Fig. 1(c)). Finally, the fabric was taken out from the case and we used as a wrinkled sample. A sample fabric wrinkle image is shown in Fig. 2.

\section{3 Image analysis}

The experimental computer vision system consists of a personal computer, a CCD camera, and a lighting arrangement. Wrinkle images were captured by a CCD camera, which was placed $600 \mathrm{~mm}$ above and perpendicular to the sample surface. The images were analyzed by the image processing system (COSMOS, LIBRARY Co. LTD.). Light sources were arranged vertically and horizontally. The light source arranged vertically was a ring type fluorescent, which was placed $600 \mathrm{~mm}$ above alongside the CCD camera. The light source arranged horizontally was a line type fluorescent, which was placed $180 \mathrm{~mm}$ away from the center of the sample at an angle of $30^{\circ}$. The intensity of illumination on samples was 28 to $40 \mathrm{~lx}$. The analysis region of each image was $350 \times 310$ pixels, corresponding to approximately $120 \times 100 \mathrm{~mm}$ of actual area in the center of a fabric, and the gray level of images was 256. A histogram equalization was operated on each input image. These images were used as wrinkle image samples for textural analysis. 


\subsubsection{Textural features}

The textural features were proposed by Haralick [12]. In this method, the relative frequencies of gray level pairs of pixels separated by a distance $d$ in the direction $\theta$ combine to form a relative displacement vector $(d, \theta)$, which is computed and stored in a matrix, referred to as gray level co-occurrence matrix $P_{d}(i, j)$ (equation (1)). This matrix is used to extract the statistical textural features.

$$
p_{d}(i, j)=\sum_{\delta} p(i, j: 1, \theta)
$$

Haralick suggested 14 features describing the two dimensional probability density function $P_{d}(i, j)$, and four of the most popular commonly used features are as follows [7, 8, 10, 11], ASM (Angular Second Moment, (2)), CON (Contrast, (3)), COR (Correlation, (4)), and ENT (Entropy, (5)). These are calculated using the equations given in Haralick [12]. ASM characterizes the homogeneity of the image, $C O N$ is a measure of contrast or the amount of local variation, $C O R$ is a measure of gray level linear dependence, and ENT is a measure of complexity of the surface and the amount of information on the image. The distance $d$ is 1 pixel line and the directions $\theta$ are $0^{\circ}, 45^{\circ}, 90^{\circ}$, and $135^{\circ}$. The textural features are averaged.

$$
\begin{aligned}
A S M= & \sum_{i=0}^{n-1} \sum_{j=0}^{n-1} p_{d, \theta}(i, j)^{2} \\
C O N= & \sum_{i=0}^{n-1} \sum_{j=0}^{n-1}(i-j)^{2} p_{d, \theta}(i, j) \\
C O R= & \frac{\sum_{i=0}^{n-1} \sum_{j=0}^{n-1}\left[i \cdot j \cdot p_{d, \theta}(i, j)-\mu_{x} \cdot \mu_{y}\right]}{\sigma_{x} \cdot \sigma_{y}} \\
& \mu_{x}=\sum_{i=0}^{n-1} i \cdot p(i), \mu_{y}=\sum_{j=0}^{n-1} j \cdot p(j) \\
E N T= & \sigma_{i=0}^{2}=\sum_{i=0}^{n-1}\left(i-\mu_{x=0}^{n-1} p_{d, \theta}(i, j) \cdot \log p_{x}(i), \sigma_{y}^{2}=\sum_{j=0}^{n-1}\left(j-\mu_{y}\right)^{2} p_{y}(j)\right.
\end{aligned}
$$

\subsubsection{Fractal dimension}

The fractal dimension is very useful and has become popular in image processing. The fractal dimension gives a feature of the roughness of a surface. Intuitionally, the larger the fractal dimension, the rougher the texture. This method has previously been applied to the study of objective evaluation for fabric wrinkles [10, 13].

The fractal dimension can be demonstrated by Equation (6):

$$
N(r) \cdot r^{D}=c
$$

Where $N(r)$ is the number of unit cells with the linear dimension defined as a unit cell length $r, C$ is some constant, and $D$ defines as the fractal dimension. This equation (6) has the solution.

$$
\log N(r)=-D \log r+\log c
$$

The fractal surface has a dimension 2 when the surface is a perfectly smooth plane and a dimension 3 when the surface is extremely rough. Therefore, the fractal dimension Fdim of wrinkle images can range from 2 to 3 .

\subsubsection{Total length of wrinkles}

The preprocessing module converts the gray level image into the bi-level image and enhances the wrinkle image with sobel filtering and thinning. And the total length $T$ of wrinkle was calculated.

\section{4 Sensory evaluation}

Fifteen experts for fabric wrinkle inspection watched 70 images of differently wrinkled fabrics. They evaluated wrinkle grades of the images using nine grades from 1 to 5 with increments of 0.5 . Additionally, they classify wrinkle states into four groups: Wrinkles formed by being worn, wrinkles formed by washing, artificial wrinkle, and unclassifiable wrinkles. They used replicas of the AATCC Test Method for evaluating wrinkle grades if necessary.

Wrinkled fabric images of $120 \times 100 \mathrm{~mm}$ in size were placed on a desk under fluorescent lights in a quiet room. The intensity of illumination on images was $700 \pm 501 x$.

\section{5 Equation for predicting wrinkle grade}

In a previous study [11], a prediction method using the degree of similarity between images was proposed, however the accuracy of the method was a little low. In this study, to improve the accuracy, a lot of textural features correlated with subjective evaluation were adopted for developing an objective equation.

For developing an objective equation to quantify the subjective value, the multiple regression analysis is often used. However, if two or more explanatory variables in a multiple regression model are highly correlated, multicollinearity problems arise and reduce the reliability of the model.

In this study, there was a possibility of multicollinearity problems occurring, because each parameter of image information was extracted from the same image and a previous study [10] has shown a good correlation between the wrinkle grades evaluated by experts and some textural features of wrinkle images. Therefore, the principal 
component regression method was applied. Principal component analysis was carried out to do the variable reduction on the explanatory variables and then a regression was run using the principle component scores as their new explanatory variables.

Principle component analysis for the parameters of image information was performed and the principle components and principle component scores were obtained. Multiple regression analysis was carried out, using the principle component score of image information as explanatory variables and the evaluated wrinkle grades as criterion variables.

\section{Results and discussion}

\section{1 Sensory evaluation}

From the results of classification of wrinkle images, 25 images were evaluated as artificial wrinkles, unclassifiable wrinkles, or wrinkles formed by being worn rather than wrinkles formed after washing. Therefore 45 images were selected form those remaining and used as wrinkle samples for analysis.

Fig. 3 shows the histogram of each grade for 45 samples. The mean grade of all samples is $3.2 \pm 0.9$, and the grade is distributed from 1.5 to 5.0. That is, the fabric wrinkles formed by the wrinkling device were distributed in each grade. From the results, it is found that the wrinkling device is effective to form various grades of wrinkles.

\section{2 Image analysis}

Table 1 shows correlation coefficients between image information values and wrinkle grades evaluated by experts. A statistically significant $(\mathrm{P}<0.01)$ correlation was observed in all the correlation coefficients. From the results of correlation coefficients, textural features of wrinkle images had a significant correlation with wrinkle grades evaluated by experts.

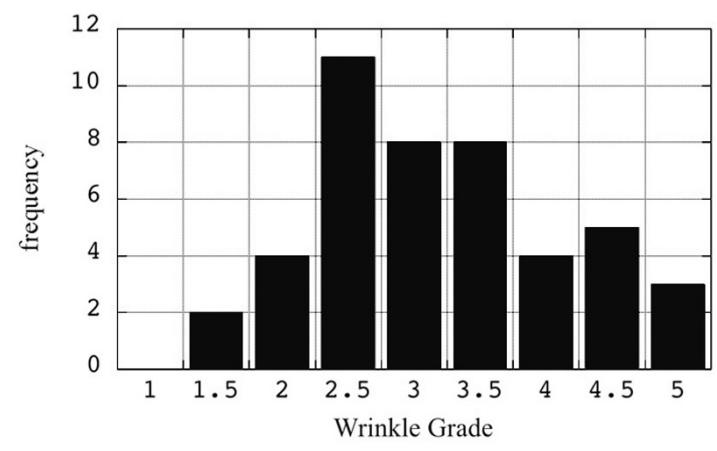

Fig. 3 Histogram of each grade for 45 samples.
Table 1 Correlation coefficient for all 70 samples between image information and wrinkle grade evaluated by experts.

\begin{tabular}{lrr}
\hline & \multicolumn{2}{c}{ Correlation } \\
& Coefficient \\
\hline$A S M(0)$ & 0.905 & $* *$ \\
$\operatorname{ASM}(45)$ & 0.884 & $* *$ \\
$\operatorname{ASM}(90)$ & 0.897 & $* *$ \\
$\operatorname{ASM}(135)$ & 0.904 & $* *$ \\
$\operatorname{CON}(0)$ & -0.554 & $* *$ \\
$\operatorname{CON}(45)$ & -0.779 & $* *$ \\
$\operatorname{CON}(90)$ & -0.865 & $* *$ \\
$\operatorname{CON}(135)$ & -0.778 & $* *$ \\
$\operatorname{COR}(0)$ & 0.528 & $* *$ \\
$\operatorname{COR}(45)$ & 0.752 & $* *$ \\
$\operatorname{COR}(90)$ & 0.859 & $* *$ \\
$\operatorname{COR}(135)$ & 0.742 & $* *$ \\
$\operatorname{ENT}(0)$ & -0.946 & $* *$ \\
$\operatorname{ENT}(45)$ & -0.918 & $* *$ \\
$\operatorname{ENT}(90)$ & -0.929 & $* *$ \\
$\operatorname{ENT}(135)$ & -0.928 & $* *$ \\
Fdim & 0.869 & $* *$ \\
$T$ & -0.943 & $* *$ \\
\hline$* * p<0.01, * p<0.05$ & \\
\hline
\end{tabular}

Fig. 4 shows the relations between each textural feature averaged by each wrinkle grade and wrinkle grades evaluated by experts. Figs. 5 and 6 show the relations between each wrinkle grade evaluated by experts and Fdim, and $T$ averaged by wrinkle grades. When the wrinkle grade was high, ASM, COR, and Fdim were large, and CON, ENT, and $T$ were small, that is, there were good correlations between the image information and the wrinkle grades. However, the correlation coefficients between wrinkle grades and $\operatorname{CON}(0)$, and $\operatorname{COR}(0)$ were lower than those of other directions, because the direction of the wrinkles formed by the device was the same as the processed direction $\left(\theta=0^{\circ}\right)$. The contrast and the linear dependence at this direction were lower than those at other directions. As the result, it was considered that differences of those features at $0^{\circ}$ between each grade were not determined.

The image information at the direction with the highest correlation coefficient was used for analysis, because the correlation coefficient at each direction $(\theta)$ in the cooccurrence features was almost equal. That is, the parameters of the co-occurrence features, $A S M(0)$, $\operatorname{CON}(90), \operatorname{COR}(90)$, and $\operatorname{ENT}(0)$ were used for analysis, and $F \operatorname{dim}$ and $T$ were also used.

Before the multivariate analysis, 45 samples were divided two groups. Group A consisted of 35 samples for developing an equation to predict the wrinkle grades and group $\mathrm{B}$ consisted of for verifying the equation. The mean grade was $3.2 \pm 0.9$ for group A and $3.2 \pm 0.9$ for group B. There was no difference in population mean of both groups. 

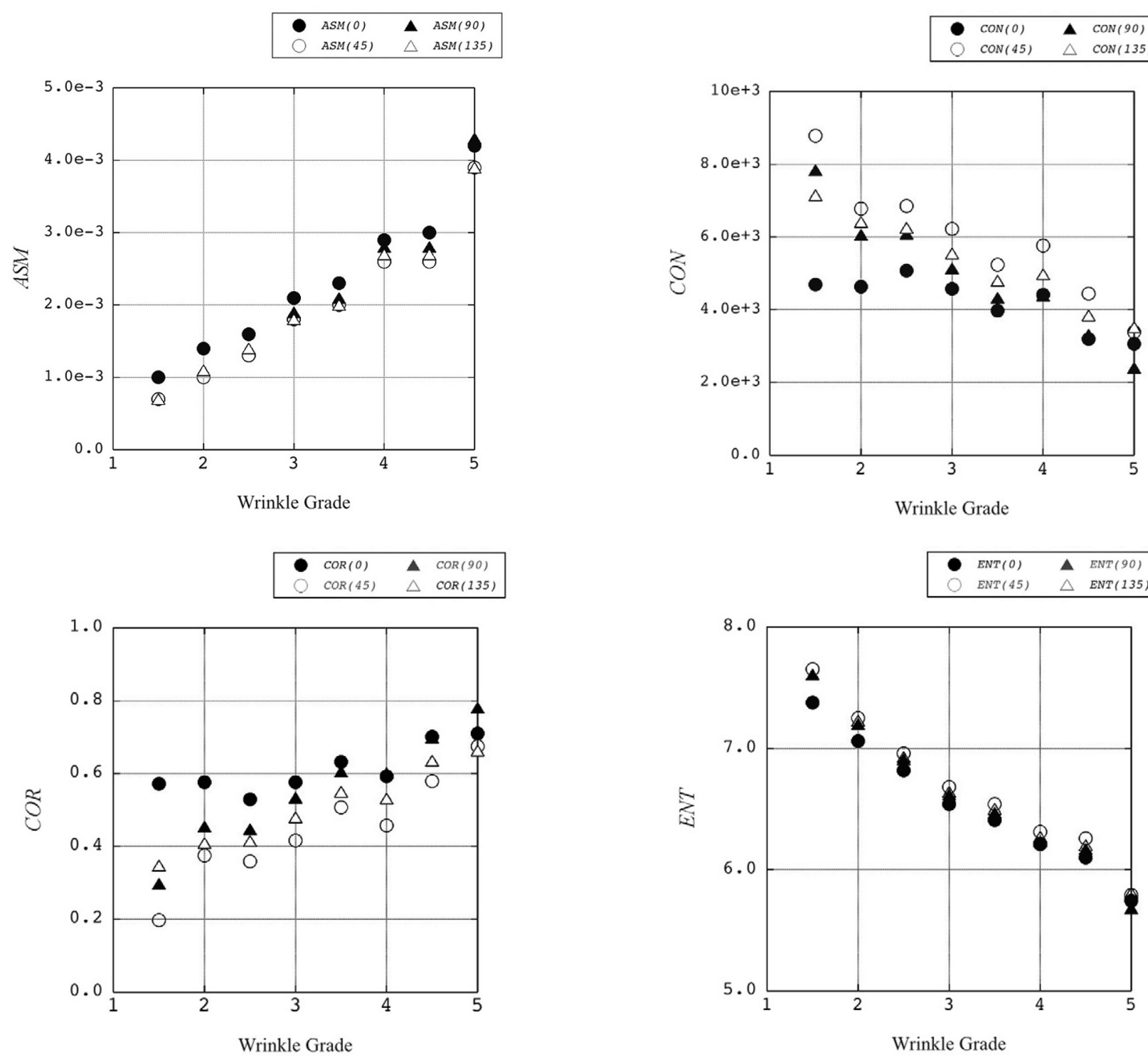

Fig. 4 Relationship between textural features (ASM, CON, COR, ENT) and wrinkle grades evaluated by experts.

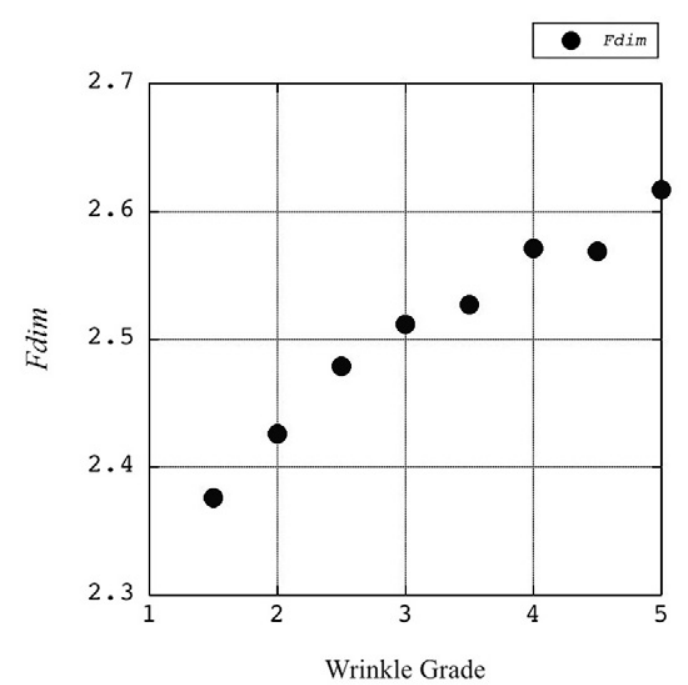

Fig. 5 Relationship between Fdim and wrinkle grades evaluated by experts.

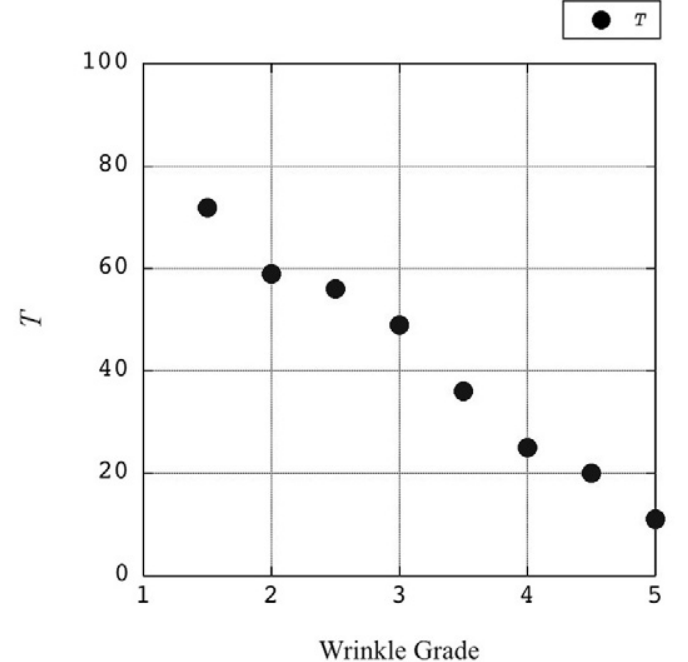

Fig. 6 Relationship between total wrinkle length $T$ and wrinkle grades evaluated by experts. 


\section{3 Objective evaluation equation for predicting wrinkle grades}

After normalizing the information parameters of images, principal component analysis was applied for the 35 samples of group A with six variables, that is $\operatorname{ASM}(0), \operatorname{CON}(90)$, COR(90), ENT(0), Fdim, and T. Principal components analysis suggested a two-dimensional solution, with the first principal component accounting for $85.7 \%$ of the variance and the other principal components descending in order from 11.4 to $0.0 \%$. The first two principal components, therefore, accounted for $97.1 \%$ of the variance. From this result, the two principal components were used for analysis. The result of principal component analysis and principal component loadings of six textural features were summarized in Table 2. Two principal component scores of each image were calculated for a multiple regression analysis.

Multiple regression analysis employing the technique of stepwise deletion was done using the two principle component scores as explanatory variables and the wrinkle grades evaluated by experts as criterion variables. As the result, we obtained the regression equation (8):

$$
Y=0.38 \times P C 1+0.02 \times P C 2+3.22
$$

The multiple R-squared of the equation was 0.940, and it is found that the accuracy of this equation is extremely high and the equation is clearly effective.

\section{4 Verifying the equation}

For verifying the obtained equation, ten other samples (group B) were analyzed and wrinkle grades of those samples were calculated with the predicting equation (8).

Fig. 7 shows the relationship between the wrinkle grades extracted by the predicting equation and the wrinkle grades evaluated by experts. The predicted grades agree well with the evaluated grades.

Table 2 Result of principle component analysis and principle component loadings of each parameter.

\begin{tabular}{lrr}
\hline & PC 1 & \multicolumn{1}{c}{ PC 2 } \\
\hline $\operatorname{ASM}(0)$ & 0.411 & 0.360 \\
$\operatorname{CON}(90)$ & -0.395 & 0.531 \\
$\operatorname{COR}(90)$ & 0.392 & -0.551 \\
$\operatorname{ENT}(0)$ & -0.426 & -0.283 \\
$F \operatorname{dim}$ & 0.402 & 0.449 \\
$T$ & -0.423 & 0.057 \\
\hline & & \\
\hline eigen value & 5.1 & 0.7 \\
principal & & \\
component & $85.7 \%$ & $11.4 \%$ \\
accounting & & \\
\hline
\end{tabular}

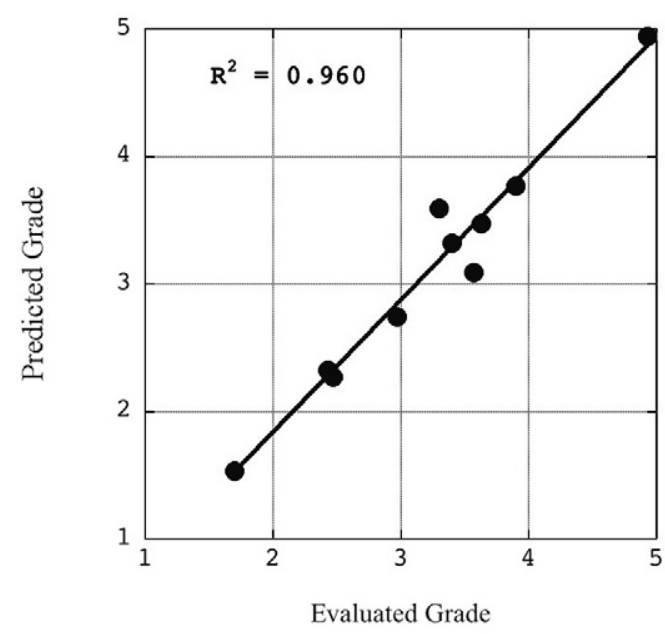

Fig. 7 Relationship between the wrinkle grades by predicting equation and the wrinkle grades by experts.

The correlation coefficient between the wrinkle grades extracted by the predicting equation and the wrinkle grades evaluated by experts was 0.98 . It is found that this equation is clearly effective for predicting the wrinkle grade.

\section{Conclusions}

The fabric images wrinkled by the experimental device were measured by the textural analysis and evaluated subjectively by experts. Using the principal component analysis and the multiple regression analysis, we proposed the objective evaluation equation for fabric wrinkle grades. The results were as follows.

(1) The experimental wrinkling device was effective to form various grades of wrinkles.

(2) From the correlation coefficients between the information values and the wrinkle grades evaluated by experts, significant correlations were observed in all correlation coefficients of each textural feature.

(3) We obtained the regression equation for predicting the wrinkle grades, using the principal component scores of image information as explanatory variables and the evaluated wrinkle grades evaluated by experts as criterion variables. The accuracy of this equation was extremely high and the equation was clearly effective.

(4) From the result of verifying the equation with other samples, the wrinkle grades extracted by predicting equation agreed well with those evaluated by experts.

\section{References}

[1] Japanese Standards Association (1998) "JISL1059-2:1998 Testing methods for crease recovery of textiles - Part 2: Evaluation of the wrinkle recovery of fabrics - Appearance method", Japanese Standards Association 
[2] The American Association of Textile Chemists and Colorists (2004) "AATCC Test Method 128-2004 Wrinkle Recovery of Fabrics: Appearance Method", The American Association of Textile Chemists and Colorists

[3] Ota N, Shibuya A (2002) J Jpn Res Assn Text End-Uses, 43, 284-293

[4] Han J, Yang M, Matsudaira M (2003) J Text Eng, 49, 1-6

[5] Su Z, Han J, Yang M, Matsudaira M (2003) Sen-i Gakkaishi, 59, 401-406

[6] Kang TJ , Cho DH, Whang HS (1999) Textile Res J, 69, 261268

[7] Mori T, Komiyama J (2002) Textile Res J, 72, 417-422
[8] Mori T, Wakida T, Endo Y (1999) Sen-i Gakkaishi, 55, 424431

[9] Matsudaira M, Han J, Yang M (2002) J Text Eng, 48, 11-16

[10] Nishimatus T, Kamijo M, Matsumoto Y, Toba E, Shibata K (2003) J Text Mach Soc Japan (predecessor journal of J Text Eng), 56, T122-T128

[11] Nishimatsu T, Oi S, Nakazawa M, Toba E, Ota K, Shibata K (2000) Sen-i Gakkaishi, 56, 348-353

[12] Haralick RM, Dinstein I, Shanmugam K (1973) IEEE Transactions, Vol. SMC-3, 610-621

[13] Kang TJ, Lee JY (2000) Textile Res J, 70, 469-475 